\title{
Optimization of Physiological Growth Conditions for Maximal Gamma-linolenic Acid Production by Cunninghamella blakesleeana-JSK2
}

\author{
S. K. Sukrutha $\cdot$ Zuzana Adamechova $\cdot$ \\ Kunder Rachana $\cdot$ Janakiraman Savitha $\cdot$ \\ Milan Certik
}

Received: 26 March 2013/Revised: 17 June 2014/ Accepted: 24 June 2014/Published online: 12 July 2014

(C) AOCS 2014

\begin{abstract}
Effects of various cultural conditions on biomass, lipid and Gamma-linolenic acid (GLA) production were investigated in the oleaginous fungus Cunninghamella blakesleeana-JSK2 isolated from soil. The GLA production was influenced by various factors such as growth condition (static and shaken), incubation time, $\mathrm{pH}$, temperature, carbon and nitrogen sources. The results indicated that optimum GLA production (21\%) was obtained when the fungus was grown under shaken condition at $120 \mathrm{rpm}$ for 6 days with optimum $\mathrm{pH}$ and temperature of 6 and $28{ }^{\circ} \mathrm{C}$, respectively. Glucose and potassium nitrate enhanced the GLA production. Urea and sucrose were poor substances for biomass, lipid and GLA production.
\end{abstract}

Keywords Gamma-linolenic acid · Submerged fermentation · Cunninghamella blakesleeana-JSK2

\section{Introduction}

There are two families of polyunsaturated fatty acids (PUFA) namely omega-3 and omega-6 fatty acids. Gamma-linolenic acid (GLA, 18:3(n-6) $\Delta 6,9,12$-octadecatrienoic acid) belongs to omega- 6 fatty acid family. It

S. K. Sukrutha $\cdot$ K. Rachana · J. Savitha $(\bowtie)$

Department of Microbiology and Biotechnology, Jnana Bharathi Campus, Bangalore University, Bangalore 560056, Karnataka, India

e-mail: drsvtj@yahoo.co.in

Z. Adamechova $\cdot$ M. Certik

Department of Biochemical Technology, Faculty of Chemical and Food Technology, Slovak University of Technology,

Radlinskeho 9, 81237 Bratislava, Slovak Republic synthesizes pro-inflammatory mediators such as series1 of prostaglandins and thromboxanes which helps in reducing inflammation. Gamma-linolenic acid is used in the treatment of rheumatoid arthritis [1], multiple sclerosis [2], schizophrenia [3], atopic eczema [4] and premenstrual syndrome [5]. It should be noted that GLA was the first PUFA to be commercially available in the market [6].

Conventionally, GLA is produced from plant sources such as borage, evening primrose, safflower and black currant. Increasing demand in the neutraceutical and pharmaceutical companies has further led to stimulated research in GLA production from microbial sources, specifically from fungi. Microorganisms are attractive candidates and possess several advantages over plants such as owing to their ease with which they can be utilized in bioprocess reactors, relatively simpler cultural conditions and nutrients for growth, no requirement of agricultural land and sunlight and consistency of the product yield.

Zygomycetous fungi such as species of Mortierella, Cunninghamella, Mucor, Rhizopus and Syzygites have been reported to accumulate lipids and GLA in their mycelia [7-11]. Thus, these lower filamentous fungi are potential candidates for the production of single cell oil containing Gamma-linolenic acid. Of late, the focus has been to maximize PUFA production by genetic manipulation, mutagenesis, response surface methodology, as well as optimizing the media conditions of the selected strain. The aim of this work was to isolate soil fungi from tropical areas and identify GLA producing fungi. Subsequently, optimizing the physiological conditions for suitable growth and maximal GLA production which would serve as models for bioprocess development. 


\section{Materials and Methods}

\section{Chemicals}

Media components used in the experiment were obtained from Merck (Bangalore, India). The carbohydrates ( $\geq 99 \%$ purity) were procured from Sigma (USA). In all our experiments, soluble starch from potato was used as the biological source. Solvents were of reagent grade. The fatty acid standards were obtained from Supelco (USA).

Isolation and Identification of Fungi from Soil Samples

One hundred soil samples were collected from different parts of Karnataka, India. One gram of each soil sample was suspended in $10 \mathrm{ml}$ of sterile distilled water, serially diluted $\left(10^{-6}\right)$ and plated on to Potato Dextrose Agar (PDA) [12] and incubated at $28^{\circ} \mathrm{C}$ for $72 \mathrm{~h}$. Individual fungal colonies were transferred to PDA slants, incubated at $28{ }^{\circ} \mathrm{C}$, assessed for purity after 4 days and stored at $4{ }^{\circ} \mathrm{C}$, till further use.

\section{Identification of Fungi}

The isolates were identified initially by observing their morphological and microscopic characteristics [12] and molecular identification was done by following the method of White et al. [13].

\section{Culture Media and Conditions}

Cunninghamella blakesleeana-JSK2 (JSK2 is the code given to the isolate chosen for further study) was grown on PDA for $24 \mathrm{~h}$ under static conditions at $28{ }^{\circ} \mathrm{C}$ before being transferred to a nitrogen limiting medium of the following composition (g/l): $\mathrm{KH}_{2} \mathrm{PO}_{4} \quad 2.5 ; \quad \mathrm{ZnSO}_{4} \cdot 7 \mathrm{H}_{2} \mathrm{O} \quad 0.01$; $\mathrm{CuSO}_{4} \cdot 5 \mathrm{H}_{2} \mathrm{O} \quad 0.002 ; \mathrm{MnSO}_{4} \cdot 0.01 ; \mathrm{MgSO}_{4} \cdot 7 \mathrm{H}_{2} \mathrm{O} \quad 0.5$; $\mathrm{FeSO}_{4} \cdot 7 \mathrm{H}_{2} \mathrm{O} 0.02 ; \mathrm{CaCl}_{2} 0.1$; yeast extract $5.0 ; \mathrm{KNO}_{3} 1.0$ and glucose 30.0 [14]. The effects of various nitrogen and carbon sources were tested by replacing potassium nitrate and glucose, respectively of the above medium with other nitrogen and carbon sources at the same concentration. The cultures were incubated at $28{ }^{\circ} \mathrm{C}$ under shaken condition (120 rpm) for 6 days.

\section{Growth Condition of Fungi}

\section{Growth Studies}

The time courses of lipid and GLA production were studied by growing the culture in $50-\mathrm{ml}$ aliquots (but results expressed as $\mathrm{g} / \mathrm{l}$ ) at $28{ }^{\circ} \mathrm{C}$ for $2,4,6$ and 8 days on a rotary shaker $(120 \mathrm{rpm})$. Cultures were harvested on respective days and washed with $100 \mathrm{ml}$ distilled water.

\section{Effect of $p H$}

The effects of $\mathrm{pH}$ ranging from 4.0 to 8.0 (by adjusting the $\mathrm{pH}$ of the medium before autoclaving using $0.1 \mathrm{M} \mathrm{HCl} /$ $\mathrm{NaOH}$ ) with one $\mathrm{pH}$ increment were used to study their effect on biomass, lipid and GLA production.

\section{Effect of Temperature}

The effects of temperature on biomass, lipid and GLA production were studied by incubating the cultures at different temperatures ranging from 24 to $32{ }^{\circ} \mathrm{C}$.

\section{Effect of Carbon Source}

The effects of various carbon sources on biomass, lipid and GLA production were studied. To find out the suitable carbon source, glucose was replaced by other carbon sources such as sucrose, starch (plant origin), maltose, Dmannitol and fructose at $30 \mathrm{~g} / \mathrm{l}$ in the basal medium.

\section{Effect of Nitrogen Source}

The effects of various nitrogen sources such as sodium nitrate, potassium nitrate, ammonium chloride, ammonium nitrate, ammonium sulfate and urea were incorporated at $1 \mathrm{~g} / \mathrm{l}$ separately in the basal medium to study their effects on biomass, lipid and GLA production.

\section{Biomass and Lipid Analysis}

\section{Cell Dry Biomass Mass Determination and Lipid Extraction}

After 6 days (144 h), cultures were harvested by filtering through muslin cloth and washed repeatedly with distilled water and lyophilized. The dried mycelial mat weight was recorded. The dried mat was made into fine powder using a mortar and pestle. The extraction of total lipids was done by following the method of Folch et al. [15]. A known amount of powdered mycelial mat was mixed with the actual volume/mass ratio of chloroform:methanol (2:1; $\mathrm{v} / \mathrm{v}$ ) and the lipids were extracted using a separating funnel. The lower fraction was collected in a beaker and allowed to evaporate to dryness. The residue containing lipids was dissolved in diethyl ether and later was transferred to pre-weighed sterilized vials, allowed to evaporate to dryness and the weights of the vials were recorded again [16]. 


\section{Fatty Acid Analysis}

The fatty acids of total lipid were analyzed as their methyl esters by gas chromatography according to Certík et al. [17]. The gas chromatograph (GC-6890 N, Agilent Technologies) was equipped with a capillary column DB$23(60 \mathrm{~m} \times 0.25 \mathrm{~mm}$, the film thickness was $0.25 \mu \mathrm{m}$, Agilent Technologies) and an FID detector (constant flow, hydrogen $35 \mathrm{ml} / \mathrm{min}$, air $350 \mathrm{ml} / \mathrm{min}, 250{ }^{\circ} \mathrm{C}$ ). Analysis was carried out with a temperature gradient $\left(130{ }^{\circ} \mathrm{C}\right.$ held for $1 \mathrm{~min}$; raised from 130 to $170{ }^{\circ} \mathrm{C}$ at a rate of $6.5^{\circ} \mathrm{C} / \mathrm{min}$; then from 170 to $215^{\circ} \mathrm{C}$ at a rate of $2.7^{\circ} \mathrm{C} / \mathrm{min}$; $215^{\circ} \mathrm{C}$ for $7 \mathrm{~min}$; from 220 to $240{ }^{\circ} \mathrm{C}$ at a rate of $2{ }^{\circ} \mathrm{C} / \mathrm{min}$; then held at $240{ }^{\circ} \mathrm{C}$ for $2 \mathrm{~min}$ ) with hydrogen as a carrier gas (flow $2.1 \mathrm{ml} / \mathrm{min}$, velocity $49 \mathrm{~cm} / \mathrm{s}$, pressure $174 \mathrm{KPa}$ ) and a split ratio of $1 / 50$ (inlets: heater $230{ }^{\circ} \mathrm{C}$, total hydrogen flow $114 \mathrm{ml} / \mathrm{min}$, pressure $174 \mathrm{KPa}$ ). The fatty acid methyl ester peaks were identified by authentic standards for a C4-C24 fatty acid methyl ester mixture (Supelco, USA) and quantified by an internal standard of heptadecanoic acid (C17:0, Supelco, USA). The fatty acid concentration was evaluated with ChemStation software B0103 (Agilent Technologies, USA). We are expressing GLA mass on the basis of the GC response data. All values were means of triplicate determinations.

\section{Statistical Analysis}

All the experiments were done in triplicate and statistical analysis was performed using SPSS software (version 11.5). Multi-variant ANOVA (post-hoc test and Scheffe test) was used to assess statistical differences. The values are given as means \pm SD (standard deviations). Levels of significance were considered at $p \leq 0.05$ unless otherwise stated.

\section{Results and Discussion}

Screening of Soil Fungi for GLA Production

A total number of two hundred fungi were isolated and among these, only Zygomycetous fungi (identified by morphological and microscopical observation) were selected to assess the oleagenicity by estimating the total lipid in general and GLA in particular (Table 1). The isolate JSK2, identified as Cunninghamella blakesleeana accumulated $41 \%$ total lipid, $21 \%$ of GLA in total fatty acids, the highest producer of GLA among the Zygomycetous fungi tested and therefore selected for further study.

\section{Molecular Identification of Cunninghamella blakesleeana-JSK2}

Molecular identification was done by sequencing the gene encoding for 18srRNA (Fig. 1). The sequence was queried in blast search National Center for Biological Information (NCBI) to find out the homology with the existing species of Cunninghamella and showed $96 \%$ similarity with Cunninghamella blakesleeana and subsequently coded as JSK2.

Growth Kinetics of Cunninghamella blakesleeanaJSK2 and GLA Production

The maximum biomass was observed on the sixth day under static conditions and on eighth day under shaking (Fig. 2a, b). The growth rate, as measured by mycelial dry weight was five times greater under shaken conditions than that was observed under static culture conditions. Cunninghamella blakesleeana-JSK2 grown under static conditions produced biomass in the form of a mycelial mat whereas the culture grown under shaking produced a mycelial mass in the form of pellets.
Table 1 Production of Dry biomass (DBM, g/l), Total lipids (TL, \%) and Gammalinolenic acid (GLA, \%) content in selected Zygomycetous fungal isolates under shaking for 6 days and other conditions are the same as given under "Materials and Methods"

Values are means $\pm \mathrm{SD}, n=3$

\begin{tabular}{clllll}
\hline S1 no & Fungal isolates & DBM $(\mathrm{g} / \mathrm{l})$ & \multicolumn{1}{l}{ TL $(\%)$} & \multicolumn{1}{l}{ GLA (\%) } & GLA (\%) in DBM (\%) \\
\hline 1 & C.nodosa-JSK1 & $2.58 \pm 0.50$ & $16 \pm 0.12$ & $18.25 \pm 0.28$ & $2.48 \pm 0.24$ \\
2 & C.blakesleeana-JSK2 & $3.00 \pm 0.28$ & $41 \pm 0.46$ & $21.09 \pm 0.59$ & $7.34 \pm 0.36$ \\
3 & Rhizopus oryzae-JSK3 & $1.59 \pm 0.36$ & $17 \pm 0.18$ & $14.54 \pm 0.61$ & $2.10 \pm 0.17$ \\
4 & Mucor spp.-JSK4 & $0.89 \pm 0.68$ & $10 \pm 0.85$ & $5.48 \pm 0.95$ & $0.46 \pm 0.22$ \\
5 & Mucor spp.-JSK5 & $5.95 \pm 0.47$ & $13 \pm 0.46$ & $6.75 \pm 0.18$ & $0.74 \pm 0.19$ \\
6 & Rhizopus spp.-JSK6 & $6.73 \pm 0.79$ & $15 \pm 0.69$ & $8.19 \pm 0.14$ & $1.04 \pm 0.09$ \\
7 & Mucor spp.-JSK7 & $4.81 \pm 0.25$ & $11 \pm 0.46$ & $6.48 \pm 0.43$ & $0.60 \pm 0.31$ \\
8 & Rhizopus spp.-JSK8 & $1.25 \pm 0.61$ & $10 \pm 0.35$ & $4.35 \pm 0.34$ & $0.36 \pm 0.25$ \\
9 & Rhizopus spp.-JSK9 & $2.56 \pm 0.94$ & $12 \pm 0.36$ & $8.24 \pm 0.63$ & $0.84 \pm 0.17$ \\
10 & Mucor spp.-JSK10 & $4.94 \pm 0.27$ & $13 \pm 0.26$ & $4.84 \pm 0.17$ & $0.53 \pm 0.22$ \\
\hline
\end{tabular}


Fig. 1 Molecular phylogenetic analysis of Cunninghamella blakesleeana-JSK2 by Internal Transcribed Spacer (ITS) $18 \mathrm{~s}$ rRNA sequence analysis

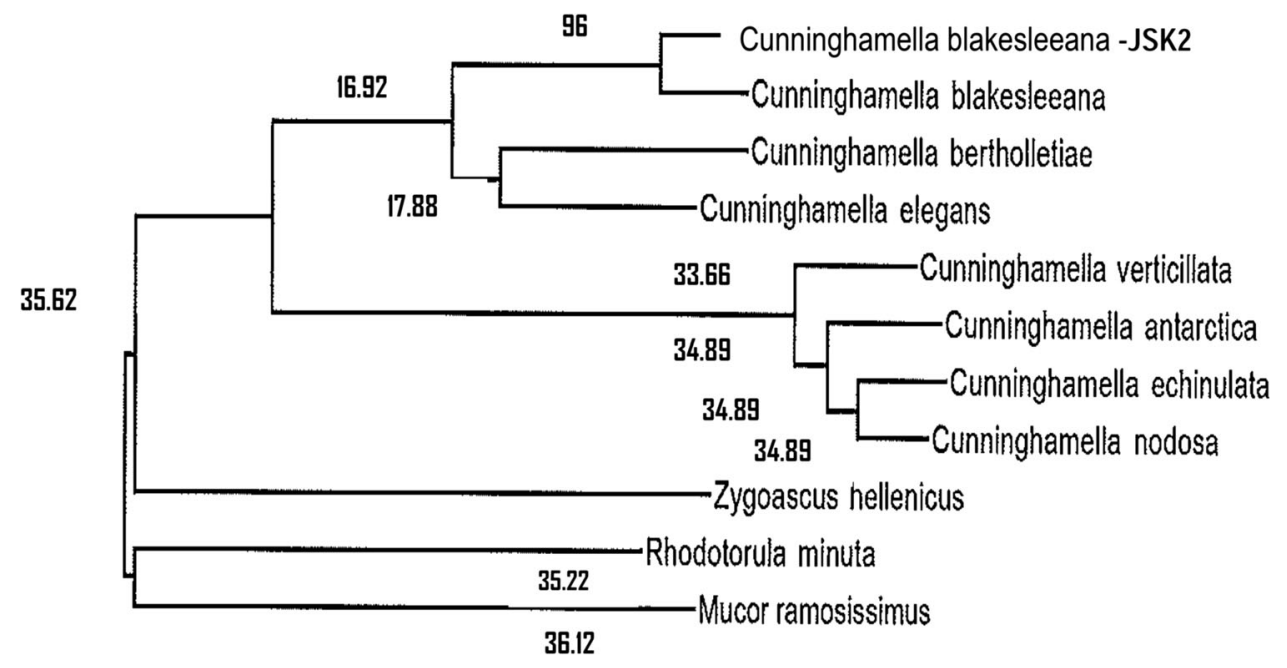

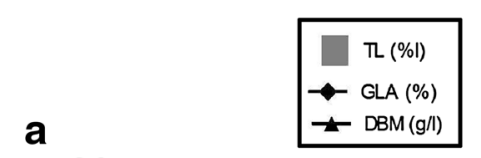
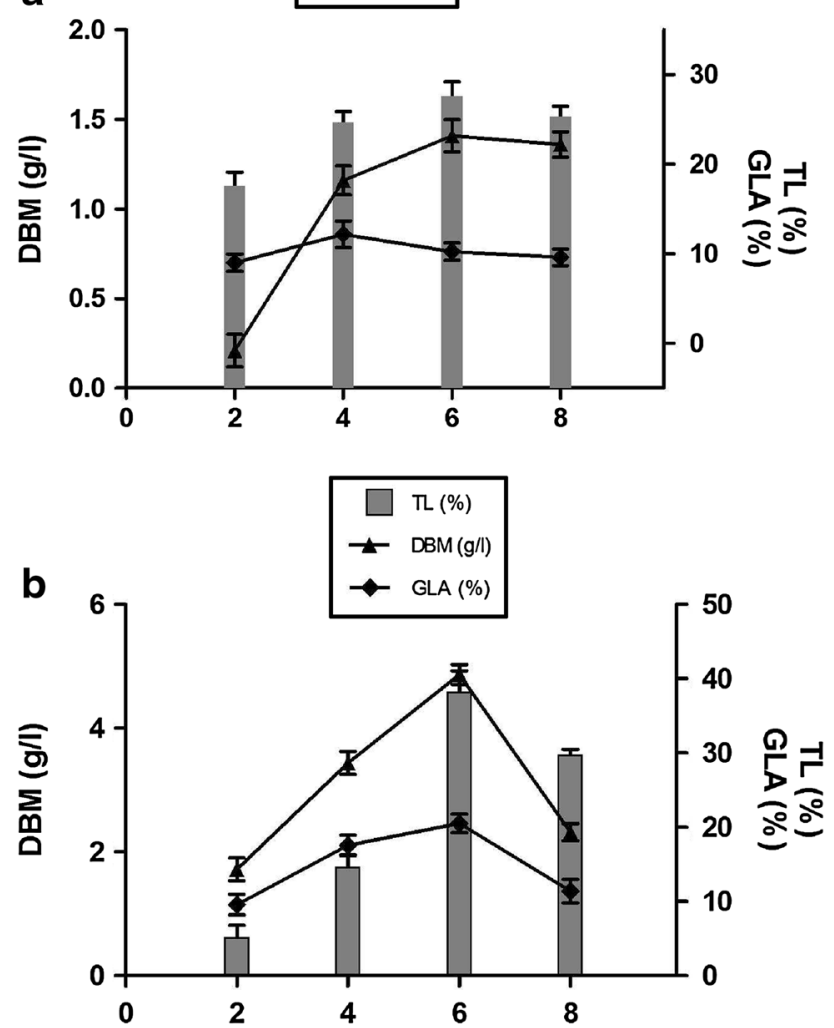

Fig. 2 a Kinetics of growth, dry biomass (DBM, g/l), total lipid (TL, \%) and Gamma-linolenic acid (GLA, \%) production in Cunninghamella blakesleeana-JSK2 under static condition for 8 days and other growth conditions are the same as given under "Materials and Methods". Values are means $\pm \mathrm{SD}, n=3$. b Kinetics of growth, dry biomass (DBM, g/l), total lipid (TL, \%) and Gamma-linolenic acid (GLA, \%) production in c. blakesleeana-JSK2 under shaken condition for 8 days and other growth conditions are the same as given under "Materials and Methods". Values are means $\pm \mathrm{SD}, n=3$
Differences in fatty acid composition of C. blakesleeana-JSK2 is seen under static and shaken condition (Table 2a, b). Lipid accumulation and GLA production under shaken condition was at a maximum on the sixth day producing $41 \%$ of total lipids and $21 \%$ of Gammalinolenic acid (Fig. 2b). Lipid accumulation under static conditions was at a maximum on the sixth day producing $28 \%$ whereas the GLA production was found to be at a maximum on the fourth day yielding $12 \%$ (Fig. 2a, b). It has been reported that lipid accumulation and GLA production decreases after 8 days due to cell disintegration [18-20].

Optimization of Temperature and $\mathrm{pH}$ for Growth and GLA Production

Temperature influences growth, lipid content and fatty acid composition in Zygomycetous fungi [21]. The maximum DBM of $6 \mathrm{~g} / \mathrm{l}$ was reached when the fungus was cultivated at $32{ }^{\circ} \mathrm{C}$ and produced the lowest amount of lipid and GLA content (Fig. 3).The optimum temperature for lipid accumulation $(26 \%)$ and GLA production $(21 \%)$ was $28{ }^{\circ} \mathrm{C}$. While the results of this study are in agreement with previously published results [22], it indicates that in fungi, the optimal temperature for growth may not necessarily be optimal for lipid production. Such parameters should be taken into account during the development of the bioprocess for large scale production.

$\mathrm{pH}$ has a profound effect on biomass, lipid and GLA production. The effect of initial $\mathrm{pH}$ was investigated from $\mathrm{pH} 4$ to 8 . Cultivation of the fungus in the range of from $\mathrm{pH}$ 5 to 6 yielded no significant difference in dry biomass. Lipid and GLA production was at a maximum when it was cultivated at $\mathrm{pH}$ 6. Biomass production and lipid accumulation was drastically reduced at extreme $\mathrm{pH}$ values(Fig 4) tested (pH 4 and $\mathrm{pH}$ 8). In Mucor sp. also, lipid 
Table 2 Relative percentage of fatty acid composition of Cunninghamella blakesleeana-JSK2 grown at different intervals of time (a) under static condition (b) under shaken for 8 days and other conditions are the same as given under "Materials and Methods"

\begin{tabular}{|c|c|c|c|c|c|c|}
\hline \multirow[t]{2}{*}{ Days } & \multicolumn{5}{|c|}{ Percentage of fatty acid composition in total lipid (\%) } & \multirow[t]{2}{*}{ GLA $(\%)$ in } \\
\hline & 16:0 & 18:0 & $18: 1$ & $18: 2$ & $18: 3(\gamma)$ & \\
\hline \multicolumn{7}{|l|}{ (a) } \\
\hline 2 & $18.03 \pm 0.12$ & $6.07 \pm 0.14$ & $42.10 \pm 0.07$ & $17.93 \pm 0.05$ & $9.01 \pm 0.11$ & $1.35 \pm 0.37$ \\
\hline 4 & $19.71 \pm 0.05$ & $12.11 \pm 0.24$ & $33.69 \pm 0.17$ & $16.21 \pm 0.15$ & $12.19 \pm 0.08$ & $2.55 \pm 0.72$ \\
\hline 6 & $18.58 \pm 0.07$ & $4.71 \pm 0.18$ & $39.68 \pm 0.26$ & $20.23 \pm 0.12$ & $10.03 \pm 0.09$ & $2.35 \pm 0.58$ \\
\hline 8 & $17.67 \pm 0.16$ & $4.94 \pm 0.06$ & $39.46 \pm 0.16$ & $19.62 \pm 0.24$ & $9.62 \pm 0.14$ & $2.07 \pm 0.86$ \\
\hline \multicolumn{7}{|l|}{ (b) } \\
\hline 2 & $16.81 \pm 0.17$ & $9.74 \pm 0.24$ & $34.15 \pm 1.15$ & $17.36 \pm 0.04$ & $9.57 \pm 0.11$ & $1.16 \pm 0.56$ \\
\hline 4 & $18.92 \pm 0.14$ & $12.11 \pm 0.08$ & $38.63 \pm 1.07$ & $14.71 \pm 0.19$ & $17.57 \pm 0.07$ & $3.23 \pm 0.62$ \\
\hline 6 & $18.36 \pm 0.18$ & $8.37 \pm 0.07$ & $27.7 \pm 1.2$ & $19.0 \pm 0.27$ & $20.48 \pm 0.13$ & $7.06 \pm 0.78$ \\
\hline 8 & $20.89 \pm 0.06$ & $6.33 \pm 0.13$ & $36.45 \pm 0.07$ & $18.0 \pm 0.21$ & $11.37 \pm 0.17$ & $1.86 \pm 0.84$ \\
\hline
\end{tabular}

[C16:0 (Palmitic acid), C18:0 (Stearic acid), C18:1 (Oleic acid), C18:2 (Linoleic acid), C18:3 ( $\gamma$ ) (Gamma-linolenic acid)]. Values are means $\pm \mathrm{SD}, n=3$

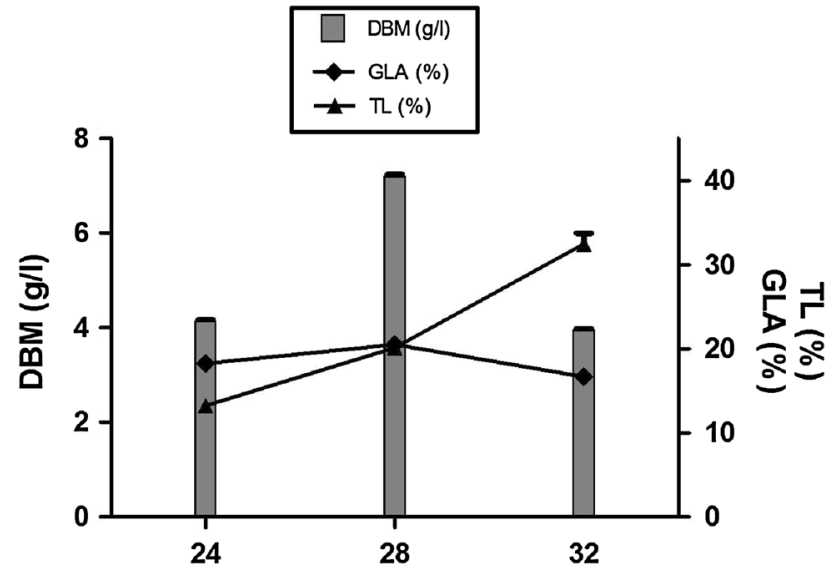

Fig. 3 Effect of temperature on dry biomass (DBM, g/l), total lipid (TL, \%) and Gamma-linolenic acid production (GLA, \%) in Cunninghamella blakesleeana-JSK2 under shaken condition for 6 days and other growth conditions are the same as given under "Materials and Methods". Values are means $\pm \mathrm{SD}, n=3$

production/accumulation was significantly reduced under sub-optimal $\mathrm{pH}$ conditions (such as, $\mathrm{pH} 4$ and $\mathrm{pH} \mathrm{8)}$ ). It is possible that interactions between media components and $\mathrm{pH}$ reduce the ability of the strain to utilize the available nutrients in the medium [22].

\section{Effect of Carbon and Nitrogen Sources}

Among the various carbon sources tested, starch yielded the highest biomass (3.4 g/l, Fig. 5). Similar results on starch were reported earlier in $M$ ramanniana var. ramanniana [23]. Total lipid production was highest in the medium supplemented with D-Mannitol (44.25\%), whereas maximum GLA production was achieved in glucose containing

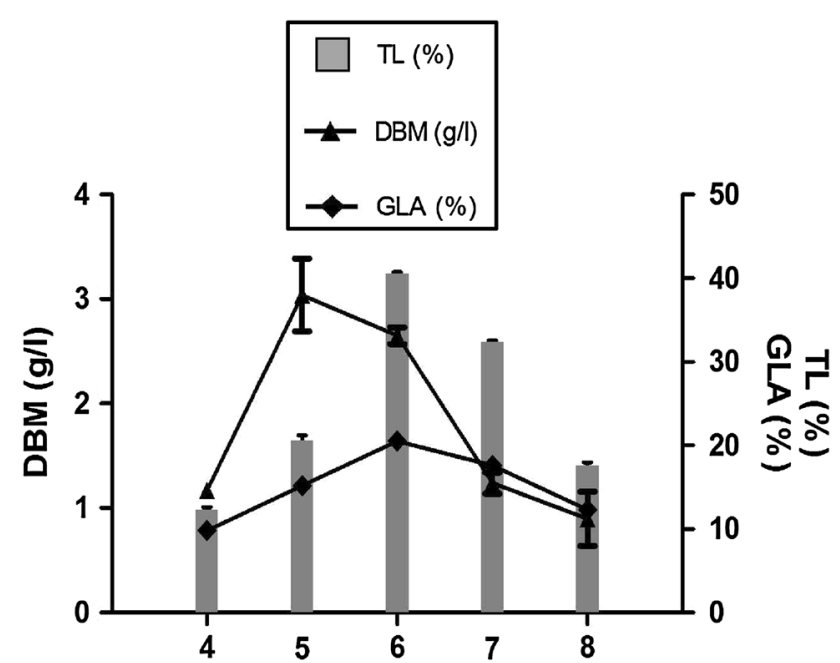

Fig. 4 Effect of initial pH on dry biomass (DBM, g/l), total lipid (TL, \%) and Gamma-linolenic acid production (GLA, \%) in Cunninghamella blakesleeana-JSK2 under shaken condition for 6 days and other growth conditions are the same as given under "Materials and Methods". Values are means $\pm \mathrm{SD}, n=3$

medium (20.5\%). This is in concordance with Mucor. sp.1b and Mucor sp. RRL001, respectively [14, 24]. Though glucose gave the highest GLA production, total lipid production was comparatively lower than on maltose, fructose and D-Mannitol (Fig. 5). Sucrose was a poor growth supporter which yielded only $1.3 \mathrm{~g} / \mathrm{l}$ dry biomass. Like sucrose, lactose have been reported as poor substance for GLA production $[14,24]$. The various sugars which supported GLA production are as follows glucose $>$ D-Mannitol $=$ fructose $=$ maltose $>$ starch $>$ sucrose. Reports suggest that a simple sugar such as glucose serves as the best carbon source for GLA production in Mucor spp., Mortierella spp. 


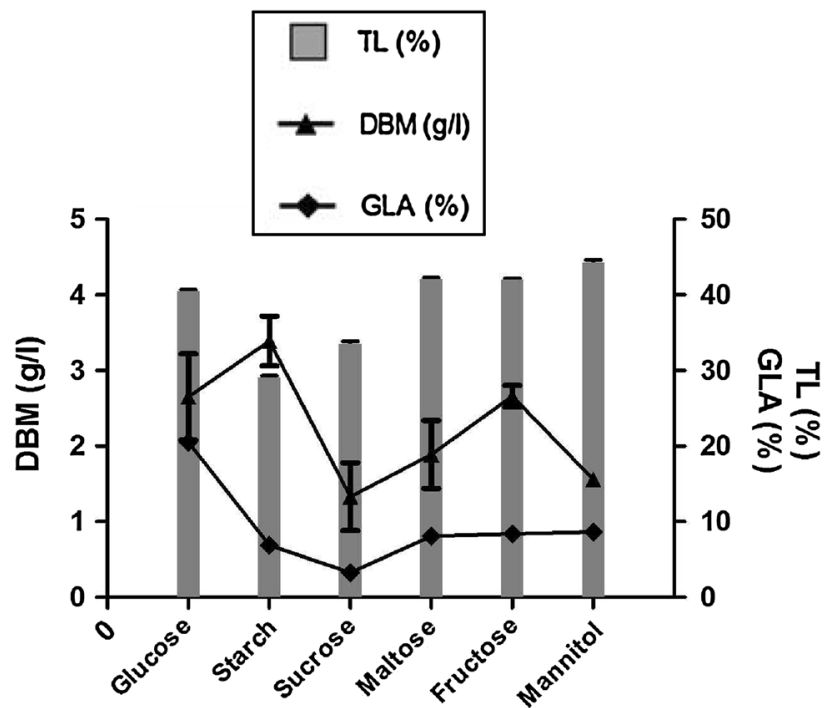

Fig. 5 Effect of different carbon sources on dry biomass (DBM, g/l), total lipid (TL, \%) and Gamma-linolenic acid production (GLA, \%) in Cunninghamella blakesleeana-JSK2 under shaken condition for 6 days and other growth conditions are the same as given under "Materials and Methods". Values are means $\pm \mathrm{SD}, n=3$

and Cunninghamella spp. [14, 23, 25]. This may be because, fungi belonging to the class Zygomycetes are generally saprophytic in nature and prefer to grow and synthesize various metabolites on simple sugars compared to complex sugars.

It has been reported that, accumulation of lipids occurs due to exhaustion of nitrogen in the medium results in cessation of cell proliferation and thus leading to the conversion of carbon into lipid [26]. Studies have indicated that nitrogen source used in the medium plays a vital role in regulating lipogenesis [27]. The influence of various nitrogen sources on biomass, lipid and GLA production was studied. Ammonium sulfate, ammonium chloride and sodium nitrate gave very nearly as much biomass as obtained with potassium nitrate $(5.7 \mathrm{~g} / \mathrm{l}$, Fig. 6). Maximum lipid production was obtained with ammonium sulfate $(36.2 \%)$. Gamma-linolenic acid production was higher in potassium nitrate supplemented medium $(21 \%)$. In the present study, least biomass production and GLA content was obtained when urea was used as nitrogen source and similar results were obtained in $M$. rouxii and $M$. spp. $1 b$ [14]. However, use of urea as nitrogen source in the growth medium enhanced biomass and GLA production of $C$. echinulata CCRC31,840 [25]. Certik et al.[27] reported maximum GLA production was obtained when sodium nitrate was used as nitrogen source in Cunninghamella echinulata. Overall, addition of potassium nitrate as a nitrogen source produced a substantial increase in GLA production over other nitrogen sources examined here.

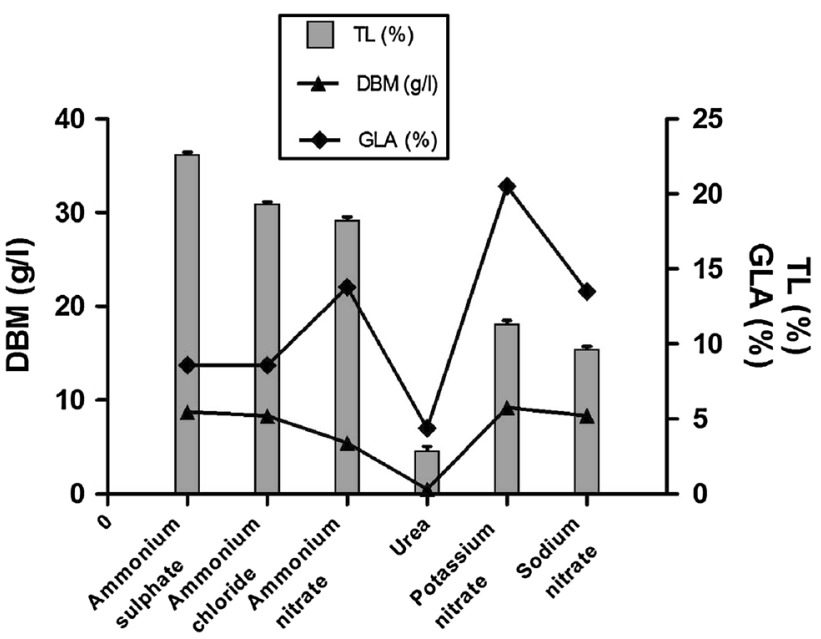

Fig. 6 Effect of different nitrogen sources on dry biomass (DBM, g/l), total lipid (TL, \%) and Gamma-linolenic acid (GLA, \%) production in Cunninghamella blakesleeana-JSK2 under shaken condition for 6 days and other growth conditions are the same as given under "Materials and Methods". Values are means $\pm \mathrm{SD}, n=3$

\section{Conclusions}

Our investigations uncovered the ability of an oleaginous fungus, $C$. blakesleeana-JSK2 isolated from soil samples to produce polyunsaturated fatty acids in general, omega- 6 fatty acid, in particular. This study also optimized physiological growth parameters which would serve as a basis for bioprocess development activities. The differences in fatty acid composition in response to different cultural parameters enable the manipulation of the production of desired polyunsaturated fatty acid. The results indicated that optimum GLA production was obtained when the fungus was grown under shaking at $120 \mathrm{rpm}$ for 6 days with an optimum $\mathrm{pH}$ of 6 and a temperature of $28^{\circ} \mathrm{C}$. Glucose and potassium nitrate enhanced the GLA production. Our present study elucidates the various physiological parameters for optimization of GLA production which would serve as a basis for future bioprocess development of therapeutically important Gamma-linolenic acid.

Acknowledgments The authors acknowledge the University Grant Council (UGC), India for funding the research project entitled "Lipid profile of endophytic fungi: Identification of suitable strain for the production of commercially important omega fatty acids (EPA \& DHA)". The work was also supported by grant VEGA 1/0975/12 from the Grant Agency of the Ministry of Education, Slovak Republic and by grants APVV-0662-11 and APVV-0294-11 from the Slovak Research and Development Agency, Slovak Republic.

\section{References}

1. Jantii J, Seppala E, Vapaatalo H, Isomaki H (1989) Evening primrose oil and live oil in treatment of rheumatoid arthritis. Clin Rheumatol 8:238-244 
2. Barber AT (1988) Evening primrose oil: a panacea. Pharmaceut J 240:723-725

3. Horrobin DF (1979) Schizophrenia: reconciliation of the dopamine, prostaglandin and opioid concepts and the role of the pineal. Lancet 1:529-531

4. Scott J (1989) Fish and evening primrose oils: gaining medical recognition. Curr Therapeut 45-46

5. Khoo SK, Munro C, Battisutta D (1990) Evening primrose oil and treatment of premenstrual syndrome. Med J Australia 153:189-192

6. Ratledge C, Wynn JP (2002) The biochemistry and Molecular biology of lipid accumulation in oleaginous microorganisms. Adv In Appl Microbiol 51:1-51

7. Fakas S, Makri A, Mavromati M, Tselepi M, Aggelis G (2009) Fatty acid composition in lipid fractions lengthwise the mycelium of Mortierella isabellina and lipid production by solid state fermentation. Biores Tech 100:6118-6120

8. Fakas S, Papanikolaou S, Batsos A, Galiotou-Panayotou M, Mallouchos A, Aggelis G (2009) Evaluating renewable carbon sources as substrates for single cell oil production by Cunninghamella echinulata and Mortierella isabellina. Biomass Bioenergy 33:573-580

9. Jangbua P, Laoteng K, Kitsubun P, Nopharatana M, Tongta A (2009) Gamma-linolenic acid production of Mucor rouxii by solid-state fermentation using agricultural by-products. Lett In App Microbiol 49:91-97

10. Kristofikova L, Rosenberg M, Vlnova A (1991) Selection of Rhizopus strains for $\mathrm{L}(+)$-lactic acid and gamma-linolenic acid production. Folia Microbiol (Praha) 36:451-455

11. Weete JD, Shewmaker F, Gandhi SR (1998) $\gamma$-Linolenic acid in zygomycetous fungi: Syzygites megalocarpus. J Am Oil Chem Soc 75:1367-1372

12. Booth C (1971) Fungal culture media. In: Booth C (ed) Methods in microbiology, vol 4. Academic Press, London

13. White TJ (1990) Amplification and direct sequencing of fungal ribosomal RNA genes for phylogenetics. In: Innis MA, Gelfand DH, Sninsky JJ, White TJ (eds) PCR protocols, a guide to methods and applications. Academic Press, San Diego, pp 315-322

14. Somashekar D, Venkateswaran G, Sambaiah K et al (2002) Effect of culture conditions on lipid and gamma-linolenic acid production by mucoraceous fungi. Proc Biochem 38:1719-1724

15. Folch JM, Lees M, Sloane-Stanley GH (1957) A simple method for the isolation and purification of total lipids from animal tissues. J Biol Chem 226:497-509
16. Savitha J, Wynn JP, Ratledge C (1997) Malic enzyme: its purification and characterization from Mucor circinelloides and occurrence in other oleaginous fungi. W J Microbiol Biotechnol 13:7-9

17. Certik M, Slavikova L, Masrnova S et al (2006) Enhancement of nutritional value of cereals with $\gamma$-linolenic acid by fungal solid state fermentations. Food Technol Biotechnol 44:75-82

18. Jang HD, Lin YY, Yang SS (2005) Effect of culture media and conditions on polyunsaturated fatty acids production by Mortierella alpina. Biores Technol 96:1633-1644

19. Yamada H, Shimizu S, Shinmen Y (1987) Production of arachidonic acid by Mortierella elongata IS-5. Agr Biol Chem 51:785-790

20. Bajpai PK, Bajpai P (1992) Review: arachidonic acid production by microorganisms. Biotechnol Appl Biochem 15:1-10

21. Sumner JL, Morgan ED, Evans HC (1969) The effect of temperature on the fatty acid composition of fungi in the order Mucorales. Can J Microbiol 15:515-520

22. Nisha A, Venkateswaran G (2011) Effect of culture variables on mycelial arachidonic acid production by Mortierella alpine. Food Bioproc Technol 4:232-240

23. Dyal SD, Bouzidi L, Narine SS (2005) Maximizing the production of $\gamma$-linolenic acid in Mortierella ramanniana var. ramanniana as a function of $\mathrm{pH}$, temperature and carbon source, nitrogen source, metal ions and oil supplementation. Food Res Inter 38:815-829

24. Ahmed SU, Singh SK, Pandey A et al (2006) Effects of various process parameters on the production of $\gamma$-linolenic acid in submerged fermentation. Food Technol Biotechnol 44:283-287

25. Chen HC, Chang CC (1996) Production of $\gamma$-linolenic acid by the fungus Cunninghamella echinulata CCRC 31840. Biotechnol Prog 12:338-341

26. Wynn JP, Hamid AA, Ratledge C et al (2001) Biochemical events leading to the diversion of carbon into storage lipids in the oleaginous fungi Mucor circinelloides and Mortierella alpina. Microbiology 147:2857-2864

27. Certik M, Megova J, Horenitzky R (1999) Effect of nitrogen sources on the activities of lipogenic enzymes in oleaginous fungus Cunninghamella echinulata. J Gen Appl Microbiol 45:289-293 